\title{
Sentinel node mapping for gastric cancer: is the jury still out?
}

\author{
Yuko Kitagawa and Masaki Kitajima \\ Department of Surgery, Keio University School of Medicine, 35 Shinanomachi, Shinjuku-ku, Tokyo 160-8582, Japan
}

The huge achievements of gastric surgeons in the last century in establishing radical surgery with extensive lymph node dissection for gastric cancer deserve unequivocal respect. Now we have to proceed to the next stage in the twenty-first century by improving postoperative function and quality of life after gastric cancer surgery without impairing long-term outcome. In this respect, the sentinel node ( $\mathrm{SN}$ ) concept may provide a breakthrough as a novel diagnostic tool. The first possible sites of metastasis along the route of lymphatic drainage from the primary lesion are known as SNs, and these are detectable using injection of dyes or radioactive tracers. After Morton et al. demonstrated the concept of SN initially in a feline model and later in a clinical study involving patients with malignant melanoma [1], the clinical impact of the SN concept in surgical oncology for various solid tumors has been a vigorously debated topic. Although we have to wait to see the results of ongoing multicenter prospective randomized control trials for SN biopsy, less invasive, modified surgical approaches are already being employed in breast cancer and melanoma. The applications of the SN concept for gastrointestinal (GI) malignancies were not recognized until the late 1990s because of the multidirectional and complicated lymphatic flow from the GI tract. However, the past 5 years have seen the recognition of several studies supporting the validity of the SN concept for GI cancers [2-5].

Despite the multidirectional and complicated lymphatic flow from gastric mucosa, the anatomical situation of the stomach is relatively suitable for SN mapping in comparison with organs such as the esophagus and rectum, embedded in enclosed spaces. In particular, clinically T1N0 gastric cancer seems to offer opportunities to modify the therapeutic approach. From the data reported in the literature, micrometastasis tend to be

Offprint requests to: Y. Kitagawa limited to within the sentinel basins in cT1N0 gastric cancer. Sentinel basins are therefore good targets of selective lymphadenectomy for cT1N0 gastric cancer in patients who are at potential risk of micrometastasis [6]. Furthermore, laparoscopic local resection is theoretically feasible for curative treatment of SN-negative early gastric cancer [7]. In Japan, clinical applications of this novel, minimally invasive approach have had great impact on patient care for gastric cancer because more than half of gastric cancer cases treated in major institutions belong to this category. Since 2000, several reports of single institutional experiences emphasizing the validity and clinical usefulness of SN mapping for gastric cancer have been published [5-11]. However, the introduction of this technology into actual standard patient care for gastric cancer requires considerable caution, because there is a potential risk of negatively affecting long-term survival due to false negative SN status results.

In this issue of Gastric Cancer, Isozaki and colleagues present the results of the first multicenter clinical trial of SN mapping for gastric cancer using a dye-guided method [12]. Although the patient population enrolled in the study is limited, several important messages can be obtained. This study was designed to investigate the feasibility of SN mapping for gastric cancer, using the dye-guided method as a simple method that can be conducted even in community hospitals without any special equipment. Although the false negative rates reported in the study ( $29 \%$ in all T1, $44 \%$ in all T2) were disappointing with regard to future clinical applications to change patient care for gastric cancer, the data of this study must be interpreted very carefully. The most serious limitation of the study was the inclusion criteria of participating institutions in terms of previous experience with SN mapping for gastric cancer. In the field of breast cancer surgery, the significance of the technical learning curve has already been clearly demonstrated [13]. Cox et al., reporting on breast cancer, suggested 
that experience of 30 cases with a detection rate of $90 \%$ or more should be the minimal requirement for participating institutions in a multicenter study of SN mapping. In the present study by Isosaki et al., the learning phase was unclear in almost all participating institutions. Consequently, the relatively high false negative rate demonstrated in the study may reasonably be attributed to technical errors in their initial learning phase. Despite these study-design limitations, the false negative rate in the $\mathrm{T} 1$ and $\mathrm{sN} 0$ patients $(11 \%)$ was compatible with previous single institutional reports. These data hold out the hope that dye-guided SN mapping might be feasible for $\mathrm{T} 1 \mathrm{sN} 0$ patients after establishing a standard procedure and completing the learning phase, in conventional open surgery. Unlike in other GI malignancies such as esophageal and rectal cancer, the dye-guided method is applicable to gastric cancer particularly in open surgery, in which mobilization of the stomach and real-time observation are feasible [6]. Although there are several limitations to the dye-directed method, such as rapid transit and blind sites in dense fat, blue dye is generally appropriate for visualizing lymphatic vessels. There still are several matters of debate concerning actual procedures, such as types of dye, injection routes (submucosal or subserosal), volume of tracer, and observation time. Generally, technical factors affect the results of lymphatic mapping for gastric cancer in the dye-guided method. After establishment of an optimal procedure, dye-guided SN biopsy of gastric cancer might have future potential clinical applications in open surgery.

However, we would like to post a special alert to those trying to apply this technique in laparoscopic surgery, which at present appears to be the final goal of SN mapping for gastric cancer as a minimally invasive approach. First of all, a feasibility study in each institutional experience in open surgery is essential before proceeding to laparoscopic feasibility study. Several remaining issues must be solved before laparoscopic application of this technology. Fast transit of blue dye (5-15 min for diffusion) is a negative factor for dyeguided laparoscopic detection of multidirectional SN of gastric cancer. Technical errors using the single mapping-agent approach are reduced by using combined approaches for lymphatic mapping. The radioguided method allows us to confirm by gamma probing the complete harvesting of SNs with multidirectional and widespread distribution, while the dye procedure enables us to perform real-time observation of the visualized lymphatic vessels. Therefore, we recommend a combination of dye- and radio-guided methods for systematic lymphatic mapping of gastric cancer. However, radio-guided SN detection is a highly advanced technology available only in specialized institutes, because special equipment and strict regulation of safety are required. Furthermore laparoscopic gamma probing is still under development owing to several technical and instrumental limitations. Currently available rigidtype laparoscopic gamma probes are fixed by the entry trocar, restricting freedom to search for $\mathrm{SNs}$ and to avoid the shine-through effect from the injection site. Furthermore, the sensitivity of intraoperative histological examination of SN is not yet satisfactory [14]. Intraoperative utilization of real-time reverse transcription-polymerase chain reaction (RT-PCR) to detect molecular metastasis is still under development. Moreover, clinical relevance of micrometastasis and isolated tumor cells are still unclear. Although SN mapping combined with laparoscopic minimally invasive surgery seems to be a promising tool for early gastric cancer, we have to solve these remaining issues with great care. As was the case in breast cancer surgery, surgeons should be very careful about media-hyped patients requesting SN mapping outside clinical trials or validated training programs.

Two major large-scale clinical trials of SN mapping for gastric cancer in conventional open surgery have been commenced in Japan. The Gastric Cancer Surgical Study Group of the Japan Clinical Oncology Group (JCOG) organized a multicenter prospective study of SN mapping by dye-guided subserosal injection of indocyanine green. The Japan Society of Sentinel Node Navigation Surgery (SNNS) study group also is conducting a multicenter prospective trial for SN mapping by combined blue dye and radioactive tracer injection. The results of these clinical trials will indicate the next direction of the field. If the JCOG study yields favorable results in terms of the false negative rate, the dyeguided method will be used in routine practice in many institutes for conventional open surgery. Should the JCOG study not yield favorable results, we must consider the introduction of a radio-guided method or further technical improvements even in open surgery. Even if the SNNS study were to demonstrate an acceptable detection rate and low false negative rate, we should conduct feasibility studies on laparoscopic SN mapping for gastric cancer as a next step. As mentioned above, there is a discrepancy between the theoretical validity of the SN concept in gastric cancer and clinical feasibility. Within the next few years, we hope to obtain the jury's definitive verdict.

\section{References}

1. Morton DL, Wen DR, Wong JH, Economou JS, Cagle LA, Storm FK, et al. Technical details of intraoperative lymphatic mapping for early stage melanoma. Arch Surg 1992;127:392-9.

2. Saha S, Nora D, Wong JH, Weise D. Sentinel node mapping in colorectal cancer-a review. Surg Clin N Am 2000;80:18119. 
3. Kitagawa Y, Fujii H, Mukai M, Kubota T, Ando N, Watanabe M, et al. The role of sentinel lymph node in gastrointestinal cancer. Surg Clin N Am 2000;80:1799-809.

4. Bilchik AJ, Saha S, Wiese D, Stonecypher JA, Wood TF, Sostrin $\mathrm{S}$, et al. Molecular staging of early colon cancer on the basis of sentinel node analysis: a multicenter phase II trial. J Clin Oncol 2001;19:1128-36.

5. Kitagawa Y, Fujii H, Mukai M, Kubota T, Otani Y, Kitajima M. Radio-guided sentinel node detection for gastric cancer. Br J Surg 2002;89:604-8.

6. Miwa K, Kinami S, Taniguchi K, Fushida S, Fujimura T, Nonomura A. Mapping sentinel nodes in patients with early-stage gastric carcinoma. Br J Surg 2003;90:178-82.

7. Kitagawa Y, Ohgami M, Fujii H, Mukai M, Kubota T, Ando N, et al. Laparoscopic detection of sentinel lymph nodes in gastrointestinal cancer: a novel and minimally invasive approach. Ann Surg Oncol 2001;8:86-9.

8. Hiratsuka M, Miyashiro I, Ishikawa O, Furukawa H, Motomura $\mathrm{K}$, Ohigashi $\mathrm{H}$, et al. Application of sentinel node biopsy to gastric cancer surgery. Surgery 2001;129:335-40.

9. Ichikura T, Morita D, Uchida T, Okura E, Majima T, Ogawa T, et al. Sentinel node concept in gastric carcinoma. World J Surg 2002;26:318-22.
10. Uenosono $\mathrm{Y}$, Natsugoe $\mathrm{S}$, Higashi $\mathrm{H}$, Ehi $\mathrm{K}$, Miyazono $\mathrm{F}$, Ishigami S, et al. Evaluation of colloid size for sentinel node detection using radioisotope in early gastric cancer. Cancer Lett 2003;200:19-24.

11. Hayashi H, Ochiai T, Mori M, Karube T, Suzuki T, Gunji Y, et al. Sentinel lymph node mapping for gastric cancer using a dual procedure with dye- and gamma probe-guided techniques. J Am Coll Surg 2003;196:68-74.

12. Isozaki $\mathrm{H}$, Kimura $\mathrm{T}$, Tanaka $\mathrm{N}$, Satoh $\mathrm{K}$, Matsumoto $\mathrm{S}$, Ninomiya M, et al. An assessment of the feasibility of sentinel lymph node-guided surgery for gastric cancer. Gastric Cancer 2004;7:149-53.

13. Cox CE, Pendas S, Cox JM, Joseph E, Shons AR, Yeatman T, et al. Guidelines for sentinel node biopsy and lymphatic mapping of patients with breast cancer. Ann Surg 1998;227:645-53.

14. Matsuda J, Kitagawa Y, Fujii H, Mukai M, Dan K, Kubota T, et al. Significance of metastasis detected by molecular techniques in sentinel nodes of patients with gastrointestinal cancer. Ann Surg Oncol 2004;11:250S-54S. 